\title{
Multiplexed Mass Spectrometry of Individual Ions Improves Measurement of Proteoforms and Their Complexes
}

Jared O. Kafader

Northwestern University

Rafael D. Melani

Northwestern University

Kenneth R. Durbin

Northwestern University

Bon Ikwuagwu

Northwestern University

Bryan P. Early

Northwestern University

Ryan T. Fellers

Northwestern University

Steven C. Beu

S.C. Beu Consulting

Vlad Zabrouskov

ThermoFisher Scientific

Alexander A. Makarov

ThermoFisher Scientific

Joshua T. Maze

ThermoFisher Scientific

Deven L. Shinholt

ThermoFisher Scientific

Ping F. Yip

ThermoFisher Scientific

Danielle Tullman-Ercek

Northwestern University

Michael W. Senko

ThermoFisher Scientific

\section{Philip D. Compton}

Northwestern University

Neil L. Kelleher ( $\nabla$ n-kelleher@northwestern.edu ) 
Northwestern University

\section{Method Article}

Keywords: Charge Detection Mass Spectrometry, Charge Detection, STORI Plots, Orbitrap, Individual Ion Mass Spectrometry

Posted Date: May 26th, 2021

DOl: https://doi.org/10.21203/rs.3.pex-820/v1

License: (c) (1) This work is licensed under a Creative Commons Attribution 4.0 International License. Read Full License

Version of Record: A version of this preprint was published at Nature Methods on March $2 \mathrm{nd}, 2020$. See the published version at https://doi.org/10.1038/s41592-020-0764-5. 


\section{Abstract}

Protocol for sample preparation, instrumental settings, and processing for the individual ion mass spectrometry method (I2MS) utilizing an Orbitrap analyzer.

\section{Introduction}

\section{Reagents}

\section{Equipment}

\section{Procedure}

Kafader et al., Nature Methods 2020

Kelleher Research Group \& Northwestern | Proteomics

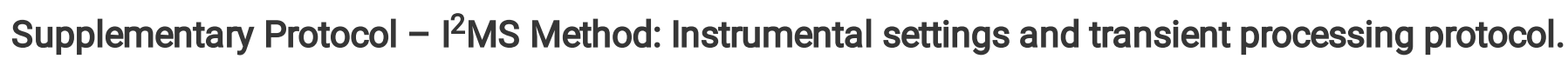

\section{Prior to Instrument Use:}

This I $^{2} \mathrm{MS}$ protocol is viable for any ThermoFisher Scientific Orbitrap instrument (QE, QE-UHMR, Fusion Lumos, Eclipse). When using these instruments access to time domain data is needed. Time domain data can also be accessed through external devices including FTMS processers purchased from companies such as Spectroswiss. The following instrumental settings need to implemented within the tune (version: 2.8, ThermoFisher Scientific) tree:

1. Spray voltages, source induced dissociation (SID), capillary temperature, and isolation window values should be optimized on a per sample basis.

2. Enhanced Fourier-transform (eFT) should be to be turned off.

3. The HCD pressure should be set to 0 .

4. For D30 Orbitraps, instrumental resolution should be set to 280,000 @ $200 \mathrm{~m} / \mathrm{z}$ with a $-5 \mathrm{kV}$ central electrode setting or 140,000 @ 200 m/z with a -1 kV central electrode setting (transients of 2 seconds in duration were used).

5. Time domain data needs to be collected in concert with .Raw file label data for each acquisition event.

6. Turn off Automatic Gain Control (AGC) and use a fixed injection time. Start with a higher inject time of $\sim 50 \mathrm{~ms}$ and adjust down as you enter the single ion regime. 


\section{During Sample Injection:}

All sample acquisitions need to be composed of 100's to 1,000's of acquisitions. As a result, direct injection needs to be utilized for stable sample ionization over extended periods of time.

1. Gain stable signal for directly injected proteins.

2. Begin with higher fixed inject times $(\sim 50-100 \mathrm{~ms})$.

3. Tune down inject times $25 \%$ and see how the charge state distribution changes.

4. Keep tuning down inject times until all signal is gone.

5. Increase inject times slightly to gain an ion distribution as that shown in the panel labeled "Step 1" in Fig. 1 workflow of the main text .

6. Begin continuous acquisition that can be monitored via Thermo Xcalibur (version: 4.0.27). Time domain data files should be kept where the .RAW file is saved.

\section{Before Processing:}

A calibration curve needs to be created composed of known charge states before unknown charge data can be collected. As each Orbitrap has differing imperfections and the trajectories of ions vary slightly from system to system, an independent calibration needs to be completed on each instrument for which $\mathrm{I}^{2} \mathrm{MS}$ will be implemented. To produce the calibration function known charge states will be isolated from well known standards. Although the calibration function will vary slightly, the procedure to collect calibration data on each charge state is the same:

1. For denatured systems prepare denatured ubiquitin, myoglobin, carbonic anhydrase, and enolase samples in a buffer containing $40 \%$ acetonitrile and $0.2 \%$ acetic acid $(\sim 0.25 \mu \mathrm{M}$ protein concentration).

Protein / Mass / Charge States / Sigma Aldrich Product

Ubiquitin / 8.5 kDa / +5 - +13 / U6253

Myoglobin / 16.9 kDa / +13 - +28 / M1882

Carbonic Anhydrase / 29 kDa / +23 - +47 / C7749

Enolase / 46.5 kDa / +35 - +65 / E6126

2. For native systems prepare native carbonic anhydrase, NIST antibody, pyruvate kinase, and GroEL samples in a buffer containing $100 \mathrm{mM}$ ammonium acetate ( 1 $\mu \mathrm{M}$ protein concentration). 
Protein / Mass / Charge States / Sigma Aldrich Product

Carbonic Anhydrase / 29 kDa / +9 - +11 / C7749

NIST Antibody / 148 kDa / +22 - +27 / NIST8671

Pyruvate Kinase / 232 kDa / +31 - +37 / 10128163001

GroEL / 801 kDa / +67 - +74 / C7688

3. To create calibration, isolate one charge states at a time with the quadrupole and collect an ion or two per acquisition from the various resolved isotopes within the charge state.

4. Approximately 30-45 minutes of data collection should be completed per charge state.

5. A sampling of charge states should be completed similar to Supplementary Figure 7.

\section{Processing:}

This process corresponds to Figure 1. Supplementary software is available with demonstration slides and dataset to illustrate how the program functions. The main focus of this processing is to determine the charge of each individual ion through its time domain data, assign a corresponding mass value and recreate the mass spectrum.

1. All collected individual ions for unknown data sets are analyzed independently.

2. The label data for each scan is read through to determine the apex peak $\mathrm{m} / \mathrm{z}$ value and corresponding frequency value of each ion signal

3. The optimal frequency is utilized to calculate the real, imaginary, and magnitude STORI slope values for each ion ${ }^{1}$.

4. Once all ion signals from every acquisition have calculated magnitude values all ion signals are ordered from minimum to maximum $\mathrm{m} / \mathrm{z}$.

5. A set number of ions from the ordered list (usually 16) are selected and their STORI slope values are averaged together.

6. If one of these STORI slopes have a value greater or less than $30 \%$ of the median STORI slope it is removed from the list or if the set number of ions do not fall within $5 \mathrm{~m} / \mathrm{z}$ of each other all ions are thrown away. 
7. The average of the remaining ions in the list is compared to the calibration function created within the Before Processing section (above) and the closest charge value from the calibration is assigned to all ions within the list.

8. This process is repeated for all ions in the unknown data set.

9. Once the charge is determined for all ions, their corresponding mass value is assigned.

10. A histogram is created in the mass domain, usually with $0.2 \mathrm{Da}$ bin sizes though use of smaller bins increases effective resolution².

\section{Troubleshooting}

\section{Time Taken}

\section{Anticipated Results}

\section{References}

1. Kafader, J.O. et al. STORI Plots Enable Accurate Tracking of Individual Ion Signals. Journal of The American Society for Mass Spectrometry 30, 2200-2203 (2019).

2. Kafader, J.O. et al. Measurement of Individual lons Sharply Increases the Resolution of Orbitrap Mass Spectra of Proteins. Analytical Chemistry 91, 2776-2783 (2019).

\section{Acknowledgements}

This work was funded by the Intensifying Innovation program from Thermo Fisher Scientific and was carried out in collaboration with the National Resource for Translational and Developmental Proteomics under Grant P41 GM108569 from the National Institute of General Medical Sciences, National Institutes of Health with additional support from the Sherman Fairchild Foundation, and the instrumentation award (S100D 025194) from NIH Office of Director. In addition, we would like to thank Luca Fornelli and Timothy K. Toby for collecting and analyzing the HEK-293 LC/MS runs utilized for our intact mass tag $\mathrm{I}^{2} \mathrm{MS}$ analysis. 